\title{
Analysis and Research of Mooring System
}

\author{
Jiahui Fan* \\ School of environment, North China Electric Power University, Baoding 071000, China \\ 591326104@qq.com
}

Keywords: Mooring system, analytic hierarchy process, multi-objective genetic algorithm.

\begin{abstract}
In this paper, the original mooring system is optimized and improved under the condition of considering the water flow and water depth. The whole system is divided into a buoy system and a mooring system. For the buoy system, the moment of gravity of the buoy at the mean sea level is analyzed. For mooring system, the anchor cable will appear elastic deformation under the action of water flow force. So the particle spring model is constructed to analyze the force of the catenary segment. Then the new wind speed critical value is obtained. Thus, the state of existence of the chain is determined. A general optimization index is set up. And the optimal solution of the optimization index is calculated according to the multi-objective genetic algorithm. Thus the design of mooring system is completed.
\end{abstract}

\section{Introduction}

The buoy system of a certain transmission node can be simplified as a cylinder with a diameter of $2 \mathrm{M}$ and a height of $2 \mathrm{~m}$. And the quality of the buoy is $1000 \mathrm{~kg}$. Mooring system is composed of a steel pipe, steel drums, heavy ball, welding anchor and special anti drag anchor. The quality of anchor is $600 \mathrm{~kg}$ and the anchor chain is provided with no file ordinary link. The steel pipe has 4 sections. Each length is $1 \mathrm{~m}$ and the diameter is $50 \mathrm{~mm}$. The quality of each steel tube is $10 \mathrm{~kg}$. The anchor angle at the end of the anchor chain should not exceed 16 degrees with the angle of the seabed. Otherwise the anchor will be towed and the node displacement will be lost. When the steel drums is vertical, equipment work best. When the tilt angle of the steel barrel is more than 5 degrees, the equipment works poor. In order to control the tilt angle of drums, the heavy ball can be hung in the links between the welding anchor and steel drums.

The design problem of the mooring system is to determine the quality of the chain type, length and weight of the ball, which make the draft depth and swimming range of the buoy and the tilt angle of the steel drums as small as possible. Data show that sea level fluctuations can be considered as a stationary and ergodic stochastic processes ${ }^{[1]}$. Therefore, it is possible to take a state to study. According to the surface equation of the water surface, roll angle and wave slope, the motion state and the draft depth of the buoy are obtained.

\section{Assumptions}

1. The wind is parallel to the plane of the sea;

2. It is hinged between steel pipe and anchor and steel;

3. It is hinged between the steel pipe and the steel pipe;

4. This paper doesn't consider up and down swing of the buoy;

5. This paper doesn’t consider viscous resistance. 


\section{Symbol Specification}

Table 1 . Symbol and its meaning

\begin{tabular}{cc}
\hline symbol & meaning \\
\hline$\eta(x, y, t)$ & wave shape function \\
$a_{i j}$ & function amplitude \\
$\xi_{i j}$ & function phase \\
$\sigma_{j}$ & wave direction \\
$w_{i}$ & function frequency \\
$k_{i}$ & wave number \\
$\sigma$ & rowing angles \\
$\varphi$ & wave slope \\
$J$ & hydraulic gradient \\
$\gamma$ & kinematic viscosity \\
$c$ & flow-resistance factor \\
$A_{y i}$ & projected area of the anchor i \\
$h_{i}$ & the depth of water at the particle $\mathrm{i}$ \\
$u_{i}$ & velocity of the depth of the anchor i \\
$l_{i}$ & the length of the anchor i \\
\hline
\end{tabular}

\section{Establishment of BP neural network model}

\subsection{Model Preparation}

\section{(1) Wave frequency}

After considering the flow force, the draft depth of the buoy changes with time. Scientists think it is the most effective way to study sea surface waves as a random wave ${ }^{[2]}$. Therefore, we can estimate the whole time frequency domain characteristics of ocean waves at any time. When analyzing the wave, the wave can be regarded as the superposition of the sine waves of wave frequency, wave height and wave direction. So the wave can be obtained

$$
\eta(x, y, t)=\sum_{j=1}^{\infty} \sum_{i=1}^{\infty} a_{i j} * \cos \left(k_{i}\left(x^{*} \cos \sigma_{j}+y^{*} \sin \sigma_{j}\right)-w_{i} t+\xi_{i j}\right)
$$

(2) Force analysis of buoy

After considering the current force, the buoy is driven by the wave to produce the rolling motion. So the classical Froude-Krylof theory is used to linearize the drag force and inertia force of the fluid acting on the buoy ${ }^{[3]}$. We can obtain the wave propagating from the origin along the square of the $\mathrm{X}$ :

$$
Y=\frac{H}{2} \cos 2 \pi\left(\frac{t}{T}-\frac{X}{L}\right)
$$

The frequency of waves is

$$
\frac{\partial Y}{\partial X}=\tan \varphi=\frac{\pi H}{L} \sin 2 \pi\left(\frac{t}{T}-\frac{X}{L}\right)
$$

When the buoy oscillates in the waves, its restoring moment is Fbuoyancy

$$
M=W \overline{g m} \sin (\sigma-\varphi)
$$




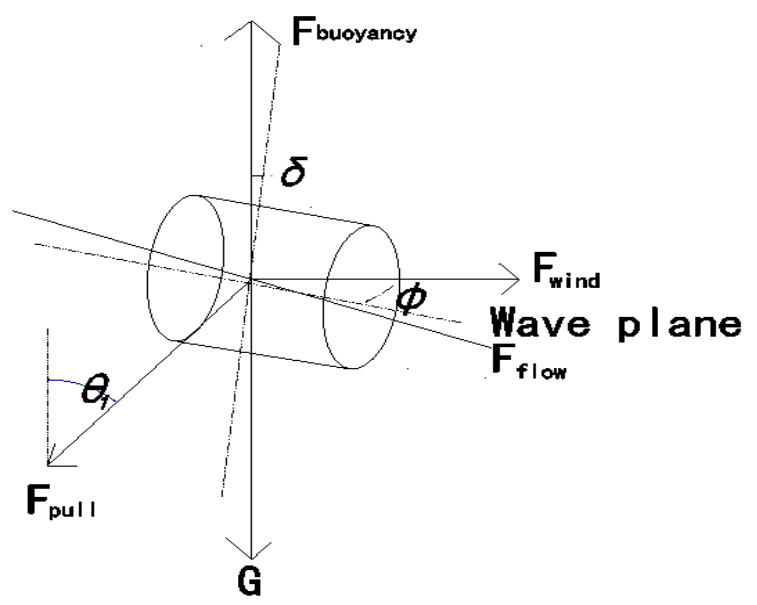

(3) Cable analysis

Fig. 1 Force analysis diagram

Because of the elastic deformation of the anchor chain under the influence of the flow force, the catenary segment of the chain is idealized as particle connected with a large number of massless spring. It is called the particle spring model ${ }^{[4]}$.

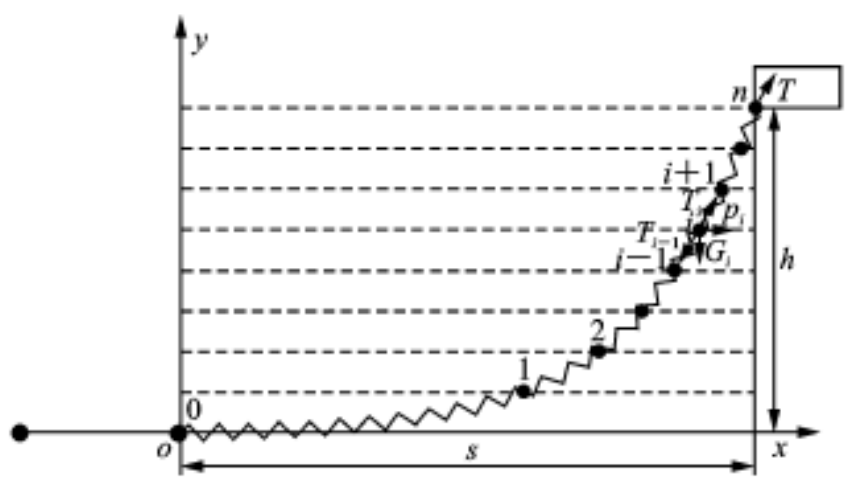

Fig. 2 Mass spring model

\subsection{Model establishment}

According to the rule of uniform flow in the open channel of binary open channel uniform laminar flow ${ }^{[5]}$, The flow rate of a water layer with a height of y can be expressed as

$$
u(y)=\frac{g J}{\gamma}\left(H y-\frac{y^{2}}{2}\right)
$$

The water flow force at anchor chain of chain $\mathrm{i}$ is as follows

$$
p_{i}=\frac{1}{2} \rho c u_{i}{ }^{2} A_{y i}
$$

The original length of the $\mathrm{i}$ anchor is

$$
l_{o i}=l_{i+1}-l_{i}=h_{i+1} \sqrt{1+\frac{2 T_{b}}{q h_{i+1}}}-h_{i} \sqrt{1+\frac{2 T_{b}}{q h_{i}}}
$$

The weight of the $\mathrm{i}$ anchor is

$$
G_{i}=q l_{o i}
$$

According to the force analysis diagram, we can obtain the static equilibrium equation

$T \sin \alpha=T_{0} \sin \alpha_{0}+q \sum l_{\text {on }}=q \sum l_{\text {on }}=q l$

$T \cos \alpha=T_{0} \cos \alpha_{0}+\sum p_{n}=T_{b}+\frac{1}{2} \rho c \sum u^{2}{ }_{n} A_{y n}$

The tension of the $\mathrm{i}$ anchor is 


$$
T=\sqrt{(q l)^{2}+\left(T_{b}+\frac{1}{2} \rho c \sum u_{n}^{2} A_{y n}\right)^{2}}
$$

After the deformation of the catenary suspension section, the horizontal projection length is

$$
s=\sum_{i=1}^{n} l_{d i} \cos \alpha_{i}=\sum_{i=1}^{n}\left[l_{o i}\left(1+\frac{T_{i}}{E A}\right) \frac{T_{b}+\frac{1}{2} \rho c \sum u^{2}{ }_{i-1} A_{y i-1}}{T_{i}}\right]
$$

Then set the target of optimization index. The 3 optimal quantities (the draft depth and swimming range of the buoy and the tilt angle of the steel drums) are analyzed hierarchically and solved by the model. According to the results obtained, each parameter value of the anchor chain and its target optimization index can be obtained. Then the pros and cons can be inferred.

\section{Example calculation}

Due to the influence of tide and other factors, the measured water depth in the distribution sea area is between $16 \mathrm{~m} \sim 20 \mathrm{~m}$. The maximum speed of the sea water at the cloth point can reach $1.5 \mathrm{~m} / \mathrm{s}$ and the maximum wind speed can reach $36 \mathrm{~m} / \mathrm{s}$.

First of all, the analytic hierarchy process of the three optimal quantities is carried out

Table 2. Three optimal quantities weights

\begin{tabular}{cccc}
\hline optimal quantities & tilt angle & the draft depth & swimming range \\
\hline weights & 0.47460 & 0.16811 & 0.35729 \\
\hline
\end{tabular}

After three optimal quantities obtained, the optimization index of the target is calculated by using multi-objective genetic algorithm

Table 3. Corresponding index of anchor chain

\begin{tabular}{cccccc}
\hline $\begin{array}{c}\text { ancho } \\
\mathrm{r}\end{array}$ & tilt angle & the draft depth & swimming range & cable shape & \multicolumn{2}{c}{ inclination of each pipe } \\
\hline II & 3.55 & 1.334 & 11.452 & 4.25 & 9.139 .259 .389 .41 \\
\hline
\end{tabular}

The minimum target optimization index is 69.37. Draft depth is $1.334 \mathrm{~m}$ and the swimming area is $11.452 \mathrm{~m}$ and tilt angle is $3.55^{\circ}$. The angle between the anchor and the sea floor is $4.25^{\circ}$.

\section{Conclusion}

In this paper, the flow force is considered. Through the stress analysis of each splitting part, the research process is simplified. The force of the water is converted into the tension of the spring confrontation point. It is convenient for force analysis without losing accuracy. According to the multilevel analysis of the optimized component, the weights are determined. And the complex system is decomposed so that it is easy to accept. Then the optimal solution is obtained by multi-objective genetic algorithm. The parameters satisfying the design requirements of the mooring system can be obtained according to the known conditions by this model. It can also compare the pros and cons of different design alternatives. It is in line with the actual situation and has relatively obvious advantages and promotion value.

\section{References}

[1] Long Yu, Jiahua Tan. Multi component mooring line quasi-static method optimization based on [J]. Ocean engineering, 2005, 01: 69-73.

[2] Shengchang Wen. Wave theory and calculation principle. [M]. Science Press, 1984.

[3] Yi Rong, Huiqiang Zhang, Xilin Wang. Mechanism analysis of gas solid two phase turbulent jet particles on gas phase turbulence [J]. Journal of Engineering Thermophysics, 2007, 05: 791-794. 
[4] Yunhe Chen, Jing Zhao. Calculation method of chain force considering flow force [J]. Journal of PLA University of Science and Technology (NATURAL SCIENCE EDITION), 2016, 02: 116-120. 